\title{
ORIGINAL RESEARCH \\ Altered Fetal Cerebral and Cerebellar Development in Twin-Twin Transfusion Syndrome
}

T. Tarui

O.S. Khwaja

J.A. Estroff

J.N. Robinson

M.C. Gregas

P.E. Grant
BACKGROUND AND PURPOSE: Neurodevelopmental disability is common in twins with TTTS in utero; however, the responsible neuropathology remains uncertain. We proposed to document the frequency of brain abnormalities on clinical fetal MR images and to determine if quantitative fetal brain biometric analysis in twin fetuses with TTTS was different from those in healthy control fetuses.

MATERIALS AND METHODS: We reviewed the fetal brain MR images of 33 twin pairs with TTTS clinically evaluated in our institution. Eighteen fetal MR images of "healthy" twins with TTTS were further studied with biometric analysis in comparison with GA-matched singleton fetuses to detect quantitative differences in brain growth and development.

RESULTS: A higher incidence of anomalies (11/33, 33.3\%) was found than previously reported. The most frequent abnormality was ventriculomegaly $(7 / 11,63 \%)$ in both donor and recipient. In "healthy" twins with TTTS, biometric analysis revealed persistently small measurements (cBTD, CMT, TCD, and VAPD) in the donor cerebrum and cerebellum in comparison with their recipient cotwin and healthy control fetuses. These differences were preserved when normalized by CBTD.

CONCLUSIONS: Our findings show that significant brain abnormalities are common in TTTS. In addition, diffuse subtle abnormalities are also present in normal-appearing donor fetal brains that cannot be solely explained by overall growth restriction. Such subtle fetal brain anomalies may explain the high incidence of poor long-term neurodevelopmental outcomes of survivors, and they need to be further investigated with more sophisticated quantitative fetal imaging methodologies.

ABBREVIATIONS: BPD = biparietal diameter; $\mathrm{CBTD}=$ cerebral bitemporal distance; $\mathrm{CMT}=$ cerebral mantle thickness; $\mathrm{GA}=$ gestational age; $\mathrm{PVL}=$ periventricular leukomalacia; $\mathrm{TCD}=$ transverse cerebellar diameter; TTTS = twin-twin transfusion syndrome; VAPD = vermian anteroposterior diameter
$\mathbf{T}$ winning is associated with an increased risk of neurodevelopmental impairment, especially when TTTS occurs. ${ }^{1}$ TTTS is a serious progressive fetal pathology that occurs in $10 \% \sim 20 \%$ of monochorionic diamniotic twin pregnancies. ${ }^{2}$ In TTTS, disproportionate intertwin transfusion via placental anastomoses causes circulatory depletion in 1 twin (donor) and overload in the other (recipient). ${ }^{3}$ This cardiovascular instability may trigger further systemic changes that result in multiple organ injury, including the brain, in both twins in utero during the second and third trimesters. In twins, TTTS is a significant cause of cerebral palsy (motor disability) and cognitive and behavioral disabilities ${ }^{4,5}$ and is a major cause of prematurity. ${ }^{6-8}$ There is a high risk of morbidity $(12 \%-$ $22 \%)^{4,9-11}$ and perinatal mortality $(30 \%)$ in survivors even after treatment.

The correspondence between underlying fetal neuropathology and postnatal neurodevelopmental outcome is not well-characterized in TTTS. Previous fetal sonographic and MR imaging studies have found various brain lesions originating antenatally, including hydranencephaly, porencephaly,

Received July 21, 2011; accepted after revision August 30.

From the Departments of Neurology (T.T., O.S.K.), Radiology (J.A.E., M.C.G.), Pediatrics (P.E.G.), and Clinical Research Program (M.C.G.), Children's Hospital Boston, Harvard Medical School, Boston, Massachusetts; Department of Obstetrics and Gynecology (J.N.R.), Brigham and Women's Hospital, Harvard Medical School, Boston, Massachusetts; and Department of Pediatrics (T.T.), Floating Hospital for Children, Tufts University School of Medicine, Boston, Massachusetts.

Please address correspondence to Tomo Tarui, MD, Department of Neurology, Children's Hospital Boston, Fegan 11, 300 Longwood Ave, Boston, Massachusetts 02115; e-mail: Tomo.Tarui@childrens.harvard.edu

http://dx.doi.org/10.3174/ajnr.A2922 polymicrogyria, other alterations in sulcation, periventricular leukomalacia, basal ganglia damage, germinal matrix hemorrhage, ventriculomegaly, and intracranial hemorrhage. ${ }^{12-14}$ The lesion type varies on the basis of the timing and mechanism of injury. These reports have demonstrated that fetal MR imaging is more accurate at detecting neurologic complications of TTTS than fetal sonography.

As with premature neonates ${ }^{15-17}$ and growth-restricted fetuses, ${ }^{18,19}$ cerebral lesions are not identified in all fetuses with TTTS, and these lesions cannot explain the whole spectrum of long-term cognitive and behavioral disabilities, which have been observed in surviving children without apparent cerebral lesions. In these children, late-emerging neurodevelopmental abnormalities, such as impairment of language and learning, are increasingly recognized. We hypothesized that fetuses with TTTS may have subtle global structural abnormalities such as gray and white matter volume reduction and altered growth. However, without focal lesions recognizable by MR imaging, these brains are visually perceived as "normal."20,21 To determine whether subtle global changes existed, we retrospectively reviewed and performed MR imaging biometric analysis on the fetal MR images of patients with TTTS seen at our institution between 2003 and 2009.

\section{Materials and Methods}

\section{Fetal MR Imaging}

We reviewed the fetal MR images of 33 monochorionic diamniotic twin pregnancies with a diagnosis of TTTS. All cases were referred to the Advanced Fetal Care Center at the Children's Hospital Boston 

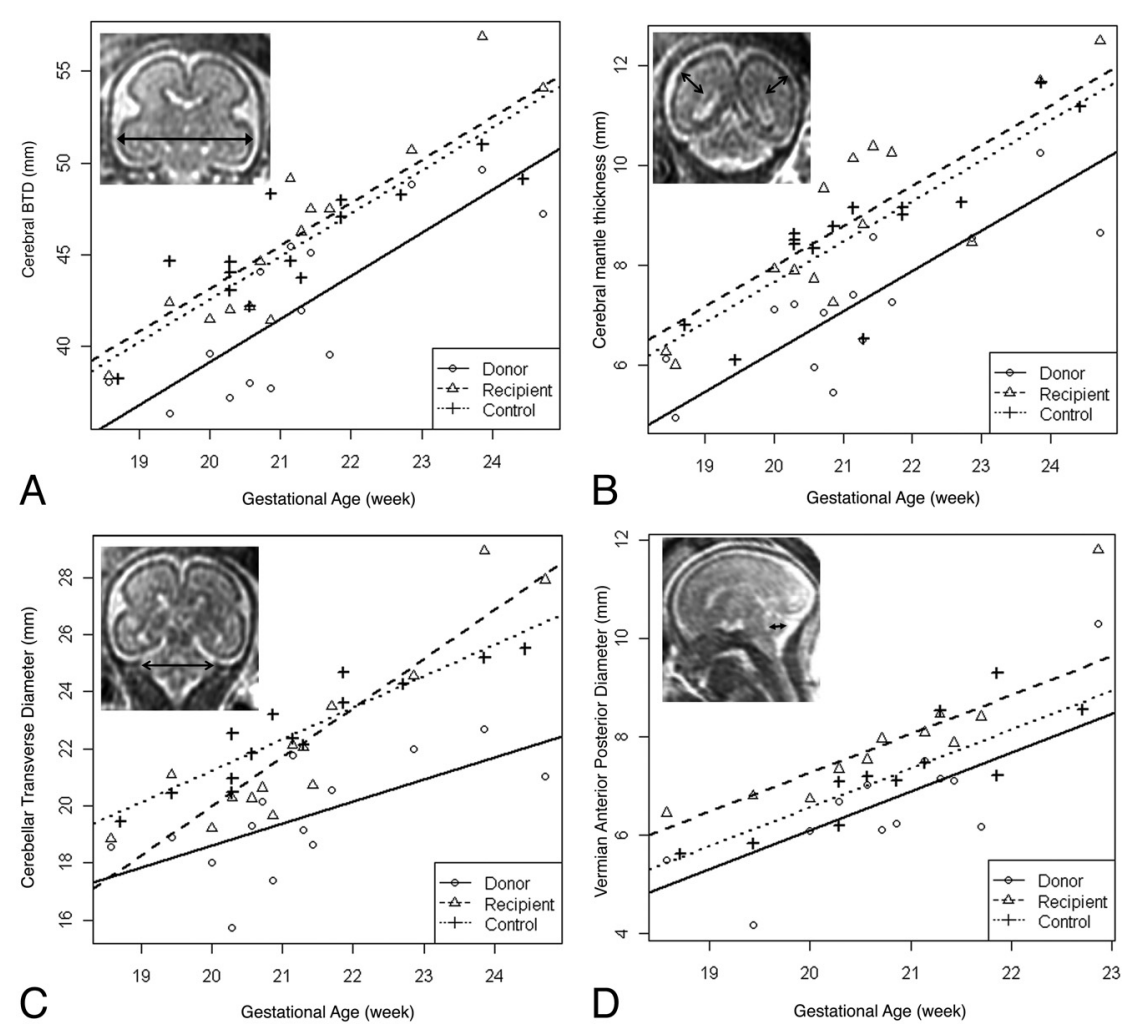

Fig 1. Growth curves of biometric parameters. Measurements of cBTD $(A)$, CMT $(B)$, TCD $(C)$, and VAPD $(D)$ are plotted for donor (circle), recipient (triangle), and control fetuses (cross). Growth curves of each parameter are modeled and illustrated for donor (solid line), recipient (broken line), and control (dotted line) fetuses. The corresponding actual measurement is illustrated in each MR image.

between January 2003 and June 2009. T1- and T2-weighted images were routinely acquired for all subjects. Diffusion-weighted images were available for 11 subjects ( 13 MR imaging studies), and fast imaging employing steady-state acquisition images was also available for 11 subjects ( $15 \mathrm{MR}$ imaging studies). All brain images for each twin fetus were evaluated by 1 pediatric neuroradiologist (P.E.G.) and 1 pediatric neurologist (T.T.). The study was approved by the Children's Hospital institutional review board.

\section{Biometric Analysis of "Normal" Twin Brains}

Seventeen twins were diagnosed as "healthy" in a gross visual review of the fetal MR images. For these twins, biometric parameters of bone $\mathrm{BPD}, \mathrm{BTD}$, lateral ventricular diameter, and CMT were measured by using the following standard methods: "Bone BPD” was defined as the greatest transverse diameter between the inner tables of the skull, and "cBTD" was defined as the greatest transverse distance between the outer surface of the (temporal lobes of) cerebrum, measured in the coronal plane at the level of the temporal horns of the lateral ventricles, as described by Garel et $\mathrm{al}^{22}$ and $\mathrm{Garel}^{23}$ and Sbarbati et al (Fig $1 A) .{ }^{24}$ The diameter of the lateral ventricles was measured in the coronal plane at the level of the atria and on an axis perpendicular to that of the ventricles.

These biometric parameters are equivalent to sonographic measurements and have been established as clinical parameters. Although sonographic bone BPD is performed in the axial plane to measure the near-end outer table to the far-end inner table of the skull, MR imaging of bone BPD has been established in the coronal plane to measure the distance between the outer surfaces of the skull. The thickness of the dorsomedial cerebral mantle was measured on the same coronal section as the lateral ventricles and was defined as the greatest distance between the wall of the ventricle and the pia on an axis perpendicular to the ventricle wall (Fig $1 B$ ). The measurement was performed until 25 weeks' GA, when developing cerebral gyration can complicate the measurement.

The size of the cerebellum was measured by using the TCD (Fig $1 C$ ) and the VAPD, as defined by Garel et $\mathrm{al}^{22}$ and Garel (Fig $\left.1 D\right){ }^{23}$ "TCD" is defined as the transverse diameter of the cerebellum in the posterior coronal plane at the level of the atria. "VAPD" is defined as the greatest distance between the median part of the roof of the fourth ventricular and the dorsal vermian surface.

\section{Statistical Analysis}

Data analysis was conducted by using the statistic software package SAS (Version 9.2; SAS, Cary, North Carolina). To examine whether the biometric fetal MR imaging measures were different between the donor and its cotwin recipient, a paired $t$ test was performed between measures of paired twins. The significance level was set at 05 .

To examine the associations between GA and biometric fetal MR imaging measures for each group of fetuses (donor, recipient twins, and control fetuses), we fit separate linear regression models for each group. We also modeled GA as a continuous variable, group as a categoric variable, and their interaction (age $\times$ group) against each biometric fetal measure. For example, the TCD was fit in a groupnested model: $\left([\mathrm{TCD}]=\beta_{0}+\beta_{1} \times[\right.$ age $]+\beta_{2} \mathrm{I}($ group $=$ donor $)+$ $\beta_{2} \mathrm{I}($ group $=$ recipient $)+[$ age $] \times \beta_{3} \mathrm{I}($ group $=$ donor $)+[$ age $] \times$ $\beta_{3} \mathrm{I}($ group $=$ recipient $)$, where $\mathrm{I}($ group $=\mathrm{A})$ is the usual indicator function that has the value of 1 if the condition in parentheses is true and is zero otherwise. Healthy controls are the reference group. We tested effect modification by including the interaction term into the mode, and we accessed its significance by using the likelihood ratio 


\begin{tabular}{lc}
\hline \multicolumn{2}{l}{ Table 1: Demographics of $\mathbf{3 3}$ twins with TTTS } \\
\hline $\begin{array}{lc}\text { Demographics } & \text { Twin Pregnancy }(n=33) \\
\text { (67 Fetuses, } 1 \text { Triplet) }\end{array}$ \\
\hline \begin{tabular}{lc} 
No. of MRI studies & 45 \\
Study no. per pregnancy & 1 to 4 \\
Maternal age range (median) (yr) & 16 to $41(30)$ \\
Presentation GA (median) (wk) & 10 to $26+4 / 7(17+3 / 7)$ \\
Initial MRI GA (median) (wk) & $15+5 / 7$ to $30+0 / 7(20+2 / 7)$ \\
Study GA range (median) (wk) & $15+5 / 7$ to $32+1 / 7(21+1 / 7)$ \\
\hline & \\
\hline Table 2: Conventional fetal MRI findings of $\mathbf{3 3}$ twins with TTS \\
\hline
\end{tabular}$\quad$ Twin Pregnancy \\
Fetal MRI Findings & $(n=33)(\%)$ \\
\hline Normal & $22(67)$ \\
Abnormal & $11(33)$ \\
Ventriculomegaly & $7(21)$ \\
Mild cortical irregularity & $3(9)$ \\
Dandy-Walker malformation & $1(3)$ \\
\hline
\end{tabular}

test. When the fit of the model was significant, the significance of group changes was tested as the difference of their slopes $\left(\beta_{3}\right)$ and their intercepts $\left(\beta_{2}\right)$. The significance level was set at .05 .

\section{Results}

The demographic data of twins with TTTS are summarized in Tables 1 and 2. Retrospective review of fetal MR images from 33 pregnancies and 65 fetuses with TTTS (ranging from $15+$ $5 / 7$ to $32+1 / 7$ weeks' GA) referred to our center revealed gross abnormalities in 11 pregnancies $(33.3 \%, 9$ donors, 2 recipients), a higher percentage than has been previously reported. ${ }^{13}$ The major abnormalities observed included bilateral or unilateral ventriculomegaly ( $7=5$ donors +2 recipients $)$, mild cortical irregularity ( 3 donors), and Dandy-Walker malformation (1 donor). No hemorrhagic or porencephalic changes were observed. One case showed increasing ventriculomegaly in the donor twin, suggesting the progressive nature of brain injury in TTTS.

\section{Biometric Analysis of "Normal" Twin Brains in TTTS}

Further biometric analyses were performed in 17 (18 brain MR images, study age range from $15+5 / 7$ to 29 weeks' GA) of 22 twin pairs with normal MR imaging findings by gross visual review. Five twin pairs were excluded from the analysis due to triplet pregnancy (1 pair), cotwin death (2 pairs), or inadequate imaging planes (2 pairs).

The cBTD was significantly smaller in donor twins compared with their cotwins (recipient twin) at all ages $(P<.00001$, paired $t$ test $)$. The donor twins had a significantly thinner cerebral mantle than their cotwins (recipient) at all ages $(P<.00001$, paired $t$ test $)$. This finding remained true after the thickness of the cerebral mantle was corrected with the CBTD, suggesting that TTTS specifically impacts cerebral mantle development disproportionate to the level of overall growth restriction (represented by cBTD) at all ages $(P<.0002$, paired $t$ test $)$.

The TCD was also compared between donor and recipient twins. The TCD in donors was significantly smaller than that of recipient twins $(P<.0001$, paired $t$ test). This finding remained true after the TCD was corrected with $\mathrm{CBTD}$, suggesting that cerebellar development is specifically affected dispro- portionally to the level of overall growth restriction at all ages $(P<.03$, paired $t$ test $)$.

No difference was seen in the ventricular diameter between the donor and recipient fetuses. The linear trajectory of cerebral growth patterns in donor, recipient twins, and control singleton fetuses was similarly linear in the second trimester.

The association between GA and the growth curves of the cBTD, cCT, TCD, and VAPD in each group (donor, recipient, and control) is illustrated in Fig 1. Each growth curve is a significant fit to the linear regression model $\left(R^{2}=0.65 \sim\right.$ 0.87 ). Therefore, during the observation period from 18 to 25 weeks' GA, there is a strong linear association between GA and the growth trajectories of the $\mathrm{CBTD}, \mathrm{CCT}, \mathrm{TCD}$, and VAPD in donor, recipient, and healthy singleton fetuses. In general, the control fetuses displayed less variability (higher $R^{2}$ value) than fetuses with TTTS. This is predictable, due to the considerably larger variation in the severity of disease.

\section{Impact on Cerebral Mantle Growth in Donor Twins}

Overall brain growth, reflected by cBTD, was significantly linearly associated with GA (Fig $1 A$ ). This growth curve was fit to a group-nested model without interaction: $[\mathrm{cBTD}]=-4.46$ $+2.35 \times[$ age $]-3.41 \mathrm{I}($ group $=$ donor $)+0.57 \mathrm{I}($ group $=$ recipient $)(P$ value $=.0001)$. This model implies that the slope of the growth curve is the same for all groups, but the intercepts are significantly different (Fig $1 A$ ). The default state of the equation describes the growth curve of control singleton fetuses. The intercept is located at -3.41 for the donor and +0.57 for the recipient. The growth curve of donor fetuses is significantly different from that of recipient and control fetuses due to significantly different intercept values $(P$ value $<.0001)$. There was no significant difference between recipient and control fetuses $(P$ value $=.53)$. This suggests that the brain development of donor twins during this observation period (early third trimester) was abnormal despite the fact that "gross" MR imaging findings appeared to be normal.

The growth curve of CMT is illustrated in Fig $1 B$. Similar to cBTD, the growth curve of CMT also fits a linear regression model without interaction: $[\mathrm{CMT}]=-8.56+0.81 \times$ [age $]-$ $1.41 \mathrm{I}$ (group $=$ donor $)+0.30 \mathrm{I}$ (group $=$ recipient $)$ $(P$ value $<.0001)$. The growth curve of donor fetuses is significantly different from that of recipient and control fetuses due to a significantly different intercept value $(P$ value $<.001)$. There was no significant difference between recipient and control fetuses $(P$ value $=.39$ ).

We tested whether the different growth curve of donor twin brains is proportional to the degree of overall growth restriction. The corrected CMT was defined as the following: $[\mathrm{CMT}] /[\mathrm{cBTD}]$. The curve of the corrected CMT fits to a linear regression model without interaction: corrected CMT = $0.019+0.008 \times$ [age $]-0.025 \mathrm{I}$ (group $=$ donor $)-0.003 \mathrm{I}$ $($ group $=$ recipient $)(P$ value $=.001)$. This model also implies that the slope of the growth curve is the same for all groups but the intercepts are significantly different. Again, the intercepts, thus the growth curve, of donor fetuses is significantly different from those of the recipient and control fetuses because of significantly different intercept values $(P$ value $=.0007)$. There was no significant difference between recipient and control fetuses $(P$ value $=.63)$. Thus, TTTS specifically impacts 
the development of the cerebral mantle in donor twins, which cannot be explained solely by overall growth restriction.

\section{Impacts on Cerebellar Growth in Both Donors and Recipients}

The growth trajectory of the cerebellum, measured by the TCD and VAPD, is illustrated in Fig $1 C,-D$. Each group has a distinct growth curve of the TCD and VAPD, which is noticeable as different intercepts. The donor twin fetuses have a persistently lower growth curve compared with recipient twins and control fetuses. The growth trajectory of TCD fits a linear regression model with interaction: $[\mathrm{TCD}]=-0.77+1.10 \times$ [age] - [age] $\times 0.33 \mathrm{I}($ group $=$ donor $)+[$ age $] \times 0.62 \mathrm{I}$ $($ group $=$ recipient $)-3.88 \mathrm{I}($ group $=$ donor $)-13.69 \mathrm{I}$ $($ group $=$ recipient $)(P$ value $<.008)$. In this model, the donor and recipient curves have significantly different intercepts and slopes. The recipient curve also has significantly different intercepts and slopes compared with those in controls. Donors and controls have the same slopes but different intercepts, indicating that the donor TCD is smaller than the control TCD. Thus, TCD growth is slower in the recipient in early development but has a faster growth rate compared with that in the donor and control. By reviewing the growth curves, we discovered that the recipient TCD has more rapid growth than the control and donor. The recipient's TCD starts from a smaller range but eventually catches up and surpasses the growth curves of controls and donors by $21 \sim 22$ weeks' GA.

In VAPD, the growth curve also fits a linear regression model $(P$ value $=.002)$ without interaction: $[\mathrm{VAPD}]=-9.25$ $+0.79 \times[$ age $]-0.47 \mathrm{I}($ group $=$ donor $)+0.70 \mathrm{I}($ group $=$ recipient) $(P$ value $=.002)$, with the same slope but different intercepts. In this model, recipient twins have a larger VAPD compared with controls and donors. In contrast, donor twins have a smaller VAPD compared with controls and recipients.

\section{Discussion}

In our gross visual review of the fetal MR images of 33 pregnancies and 65 fetuses with TTTS, $29.4 \%$ of fetuses were found to have MR imaging abnormalities, most commonly ventriculomegaly. Of 33 pregnancies, 17 were found to have normal conventional MR imaging findings for each twin. However, the biometric analyses of these "healthy" twin fetuses revealed that the cerebral mantle and cerebellum were significantly smaller in donor twins compared with recipient twins and control singleton fetuses, even when accounting for overall brain growth. In addition, the cerebellum grew more rapidly in recipient fetuses compared with donor twins and agematched control singleton fetuses.

\section{Age- and Mechanism-Dependent Manifestation of Fetal Brain Injury in TTTS}

In our study, we observed 7 cases of ventriculomegaly, 3 cases with mild cortical irregularity, and 1 Dandy-Walker malformation. In previous studies, more variable lesions were detected on neonatal sonography ${ }^{12,25}$ or fetal MR imaging, ${ }^{13}$ such as schizencephaly, polymicrogyria, or periventricular leukomalacia. ${ }^{13}$ Such difference may be caused by a relatively younger age range at the time of imaging in our study $(15+$ $5 / 7$ to $32+1 / 7$ weeks' GA; median, $21+1 / 7$ weeks' GA) compared with the previous studies (18-37 weeks' GA; me- dian, $27+0 / 7$ weeks' GA in Quarello et al, $2007^{13}$; and $18+$ $1 / 7$ to $29+3 / 7$ weeks' GA; median, $22+0 / 7$ weeks' GA in Jelin et al, $\left.2008^{14}\right)$. Lesions such as PVL and polymicrogyria may become apparent only later in gestation as further cortical growth and morphometric changes occur, or the incidence of PVL may increase with later GA. MR imaging examinations later in gestation and immediately postnatally would help to better characterize the extent of fetal brain injury in TTTS. ${ }^{13}$

Unlike other studies, we noted ventriculomegaly as the most common MR imaging abnormality in fetuses with TTTS, which is more common in the donor (5 fetuses) than the recipient (2 fetuses). Fetal ventriculomegaly is a nonspecific MR imaging finding, with many potential etiologies such as hydrocephalus, parenchymal injury, cerebral malformations, and other genetic abnormalities. ${ }^{12,13,25}$ We hypothesize that in TTTS, the ventriculomegaly is due to global factors such as systemic hypoperfusion similar to that observed in fetuses with congenital heart disease. ${ }^{26}$

\section{Validity of Biometric Analysis of the Fetal Brain}

Biometric analysis of the fetal brain is clinical standard practice, especially with obstetric sonography ${ }^{27}$ and fetal MR imaging. ${ }^{23}$ The parameters such as bone BPD, cBTD, occipitofrontal diameter, and head circumference are commonly used and are well-established as valid methods to estimate overall cerebral growth. ${ }^{22-24} \mathrm{TCD}$ and VAPD have been used as a standard measurement to estimate cerebellar growth with sonography $^{27-31}$ and MR imaging. ${ }^{23,32,33}$

Two studies reported the validity of CMT measurement in the diagnosis of fetal ventriculomegaly. Siebert et $\mathrm{al}^{34}$ first reported the validity of using the CMT as a parameter to diagnose fetal ventriculomegaly in fetopsy specimens. Loo et $\mathrm{al}^{35}$ further established the validity of this measurement in fetal ventriculomegaly by studying 10 fetuses with ventriculomegaly and 120 control fetuses. They compared the validity of measurement in 3 distinct coronal planes: the occipital region through the middle of the calcarine sulcus, the posterior frontal region through the center of the lateral fossa, and the frontal region midway between the temporal and frontal poles. ${ }^{35}$ They concluded that a single plane is sufficient to measure CMT for diagnosis of (diffuse) ventriculomegaly. We have approximated the most caudal plane of the measurement of Loo et al to be at the level of the atria, because this has been used in the measurement of ventricular diameter and TCD and is easily identifiable with MR imaging.

A limitation of our study is that we do not have measurements of actual volumes of the whole brain, cerebrum, and cerebellum, which would be more reliable measures of fetal brain growth and development. To perform reliable regional segmentation and subsequent volumetric analysis, we need to acquire higher quality fetal MR images, which we propose to do in future studies.

\section{Growth Trajectory of Biometric Parameters in the Fetal Brain}

Previous studies have found that the normal growth trajectory of fetal brain volume follows the Gompertz curve, which has a steep linear growth phase in the second trimester. ${ }^{29,36} \mathrm{Simi-}$ larly, we found a linear growth trajectory in $\mathrm{CBTD}, \mathrm{CMT}$, TCD, and VAPD during that period. This was statistically sup- 
ported by a significant fit to the linear regression model. In further testing, we were able to distinguish the growth curve of donor twins compared with recipients and healthy singleton fetuses, suggesting differences in brain growth trajectory for each group during this period (Fig $1 A-D)$.

\section{Hypoplastic Cerebrum and Cerebellum:} Neuropathophysiology of Fetal Brain Injury in TTTS

A significantly thinner cerebral mantle and smaller cerebellum in TTTS donors suggests that the underlying pathology affects broad regions of the developing brain. Possible etiologies include hypoperfusion and deprivation of oxygen and other relevant sources of nutrients through decreased systemic blood supply. Such pathology could cause destruction of, or modulations in, developmental mechanisms because the cerebral mantle and cerebellum are both in an active phase of development at these GAs and are, therefore, especially vulnerable to systemic pathology.

During the second and third trimesters, developing cerebral mantle tissue has 7 distinct histologic layers, composed of various cells, neuronal fibers (axons), and extracellular matrix, and is extremely metabolically active. ${ }^{37}$ The ventricular zone is vulnerable to injurious processes due to its extremely high proliferative activity and vulnerable supporting vasculature. $^{38,39}$ The intermediate zone is composed of tangentially running neuronal cell processes (axons), a primordia of future white matter. It contains the growing axons of major pathways, such as the thalamocortical tract, differentiating various stages of oligodendrocyte lineage. ${ }^{39}$ The subplate is a transient layer, rich in cellular and extracellular components. The neurons in this layer are generated in the very early phases of cortical neurogenesis and play a critical role in receiving synaptic input from thalamic axons, therefore establishing a temporary link between the thalamic axons and their final target zone in the future layer IV. The cells in each layer are in a high metabolic energy-consumption state by executing precisely organized roles in cerebral development such as proliferation and differentiation (morphometric and functional changes). Thus, pathologic processes affecting these highly vulnerable structures may considerably affect subsequent development and future neurodevelopmental function. ${ }^{39}$

The growth curves of each biometric measure remain parallel during the second trimester; this pattern suggests that the neuropathology of TTTS is persistent. These shifted growth curves are more likely to reflect abnormal development at the microscopic level rather than simply delayed development, given the abnormal neurodevelopmental outcomes in TTTS. ${ }^{4,5,9,11}$ It is not unusual for subtle diffuse structural abnormalities to manifest as neurodevelopmental pathology, such as learning disabilities, attention deficit disorder, and behavioral disorders (autism), in contrast to the apparent neurologic deficits associated mainly with readily recognizable focal lesions (porencephaly, cystic PVL), such as in cerebral palsy. As can be seen in premature birth survivors, these diffuse changes may be rather common and may have significant impact on long-term outcomes for surviving children with TTTS. ${ }^{15-17}$ However, future studies are needed to explore whether the differences in normal and TTTS brain connectivity and function associated with these smaller volumes better define the pathologic mechanisms of neurodevelopmental sequelae.

\section{Growth of the Cerebellum in TTTS}

In this study, the observed growth pattern of the cerebellum was more complicated than that of the cerebrum. The recipient cerebellum had a steeper growth curve (faster growth) compared with both donor and control. TCD of the recipient fetus started smaller than that of the control but eventually caught up and surpassed controls at the end of the second trimester. This growth pattern is unique to the cerebellum and was not seen in cerebral growth during this observation period. This can be partly explained by the robust growth potential of the cerebellum, which outpaces growth of the cerebrum in the second and third trimesters. Histologically, the increase in granular cells in the extragranular layer and subsequently in the internal granular layer contributes to the rapid growth of the cerebellum in late gestation. ${ }^{40,41}$ Rapid growth continues postnatally mainly by continuous growth of the internal granular layer, fiber connections, and synapse formation. ${ }^{40,41}$

The etiology for increased growth in the recipient twin cerebellum is unknown. Trophic factors might accelerate brain growth, which may be detected only in the cerebellum due to its more rapid growth. Given our increasing understanding of the significant role the cerebellum plays in neurodevelopment, these cerebellar growth abnormalities are likely to contribute to observed abnormal outcomes in the TTTS population. However, as with the cerebral differences, future studies are needed to explore the differences in normal and TTTS cerebellar connectivity and function associated with these different growth curves to better define the role of these cerebellar abnormalities in the neurodevelopmental sequelae.

\section{Conclusions}

In this retrospective study, we show visually occult but measurable differences in cerebral and/or cerebellar growth in both TTTS donors and recipients compared with controls. These abnormalities may be pertinent when we consider the reported high incidence of long-term neurodevelopmental disabilities associated with survivors of TTTS.

These findings are significant for the management of surviving children with TTTS, in that many children may not be properly diagnosed as having neuropathology if we depend solely on conventional visual inspection of MR imaging studies. Further studies will be aimed at more sophisticated methodologies to better define the underlying pathology in the TTTS population, such as regional volumetrics, cortical surface area, and cortical surface curvature measurements. In addition, diffusion measures, such as mean diffusivity and fractional anisotropy and MR spectroscopy, are likely to contribute to our understanding of associated changes in tissue composition. Sophisticated quantitative methodology and postnatal follow-up studies are of critical importance in the detection of abnormalities associated with TTTS and in identifying the need to implement early intervention for surviving children at risk.

Disclosures: Tomo Tarui-RELATED: Grant. American Academy of Neurology, ${ }^{*}$ Baby Alex Foundation, ${ }^{*}$ William Randolph Hearst Fund, ${ }^{*}$ Comments: The American Academy of Neurology is an international professional association of 24,000 neurologists and neuro- 
science professionals. The Academy granted a Clinical Research Training Fellowship Award (http://www.aan.com/go/foundation/research) to me to support my ongoing study in TTTS. Julian Robinson—UNRELATED: Royalties: UpToDate online medical resource (http://www. uptodate.com). Both the Baby Alex Foundation (http://www.babyalexfoundation.com/grants. html) and William Randolph Hearst Fund (http://www.hms.harvard.edu/spa/funding/ internal/hearst.shtml) are private foundations that support medical science researchers in the field of perinatal brain injury. The grant recipients are selected by a peer-review scientific review committee in each foundation. * Money paid to the institution.

\section{References}

1. Luu TM, Vohr B. Twinning on the brain: the effect on neurodevelopmental outcomes. Am J Med Genet C Semin Med Genet 2009;151C:142-47

2. Harkness UF, Crombleholme TM. Twin-twin transfusion syndrome: where do we go from here? Semin Perinatol 2005;29:296-304

3. Galea P, Jain V, Fisk NM. Insights into the pathophysiology of twin-twin transfusion syndrome. Prenat Diagn 2005;25:777-85

4. Lopriore E, Middeldorp JM, Sueters M, et al. Long-term neurodevelopmental outcome in twin-to-twin transfusion syndrome treated with fetoscopic laser surgery. Am J Obstet Gynecol 2007;196:231, e231-34

5. Salomon LJ, Ortqvist L, Aegerter P, et al. Long-term developmental follow-up of infants who participated in a randomized clinical trial of amniocentesis vs laser photocoagulation for the treatment of twin-to-twin transfusion syndrome. Am J Obstet Gynecol 2010;203:444, e441-47

6. Duncombe GJ, Dickinson JE, Evans SF. Perinatal characteristics and outcomes of pregnancies complicated by twin-twin transfusion syndrome. Obstet Gynecol 2003;101:1190-96

7. Lutfi S, Allen VM, Fahey J, et al. Twin-twin transfusion syndrome: a population-based study. Obstet Gynecol 2004;104:1289-97

8. Smith NA, Wilkins-Haug L, Santolaya-Forgas J, et al. Contemporary management of monochorionic diamniotic twins: outcomes and delivery recommendations revisited. Am J Obstet Gynecol 2010;203:133, e1-6

9. Banek CS, Hecher K, Hackeloer BJ, et al. Long-term neurodevelopmental outcome after intrauterine laser treatment for severe twin-twin transfusion syndrome. Am J Obstet Gynecol 2003;188:876-80

10. Habli M, Lim FY, Crombleholme T. Twin-to-twin transfusion syndrome: a comprehensive update. Clin Perinatol 2009;36:391-416, x

11. Graef C, Ellenrieder B, Hecher K, et al. Long-term neurodevelopmental outcome of 167 children after intrauterine laser treatment for severe twin-twin transfusion syndrome. Am J Obstet Gynecol 2006;194:303-08

12. Lopriore E, van Wezel-Meijler G, Middeldorp JM, et al. Incidence, origin, and character of cerebral injury in twin-to-twin transfusion syndrome treated with fetoscopic laser surgery. Am J Obstet Gynecol 2006;194:1215-20

13. Quarello E, Molho M, Ville Y. Incidence, mechanisms, and patterns of fetal cerebral lesions in twin-to-twin transfusion syndrome. J Matern Fetal Neonatal Med 2007;20:589-97

14. Jelin AC, Norton ME, Bartha AI, et al. Intracranial magnetic resonance imaging findings in the surviving fetus after spontaneous monochorionic cotwin demise. Am J Obstet Gynecol 2008;199:398 e391-95

15. Marlow N, Wolke D, Bracewell MA, et al. Neurologic and developmental disability at six years of age after extremely preterm birth. $N$ Engl J Med 2005;352:9-19

16. Allen MC. Neurodevelopmental outcomes of preterm infants. Curr Opin Neurol 2008;21:123-28

17. Johnson S, Fawke J, Hennessy E, et al. Neurodevelopmental disability through 11 years of age in children born before 26 weeks of gestation. Pediatrics 2009;124:e249-57

18. Leitner Y, Fattal-Valevski A, Geva R, et al. Neurodevelopmental outcome of children with intrauterine growth retardation: a longitudinal, 10-year prospective study. J Child Neurol 2007;22:580-87

19. Walker DM, Marlow N. Neurocognitive outcome following fetal growth restriction. Arch Dis Child Fetal Neonatal Ed 2008;93:F322-25

20. Nosarti C, Giouroukou E, Healy E, et al. Grey and white matter distribution in very preterm adolescents mediates neurodevelopmental outcome. Brain 2008; 131(pt 1):205-17

21. Soria-Pastor S, Padilla N, Zubiaurre-Elorza L, et al. Decreased regional brain volume and cognitive impairment in preterm children at low risk. Pediatrics 2009;124:e1161-70

22. Garel C, Chantrel E, Elmaleh M, et al. Fetal MRI: normal gestational landmarks for cerebral biometry, gyration and myelination. Childs Nerv Syst 2003;19:422-25

23. Garel C. MRI of the Fetal Brain. New York: Springer-Verlag; 2004

24. Sbarbati A, Pizzini F, Fabene PF, et al. Cerebral cortex three-dimensional profiling in human fetuses by magnetic resonance imaging. $J$ Anat 2004;204:465-74

25. Lopriore E, Slaghekke F, Vandenbussche FP, et al. Cerebral injury in monochorionic twins with selective intrauterine growth restriction and/or birthweight discordance. Am J Obstet Gynecol 2008;199:628 e621-25

26. Limperopoulos C, Tworetzky W, McElhinney DB, et al. Brain volume and metabolism in fetuses with congenital heart disease: evaluation with quantitative magnetic resonance imaging and spectroscopy. Circulation 2010;121:26-33

27. Altman DG, Chitty LS. New charts for ultrasound dating of pregnancy. Ultrasound Obstet Gynecol 1997;10:174-91

28. Goldstein I, Reece EA, Pilu G, et al. Cerebellar measurements with ultrasonography in the evaluation of fetal growth and development. Am J Obstet Gynecol 1987; 156:1065-69

29. Hill LM, Guzick D, Rivello D, et al. The transverse cerebellar diameter canno be used to assess gestational age in the small for gestational age fetus. Obstet Gynecol 1990;75(3 pt 1):329-33

30. Co E, Raju TN, Aldana O. Cerebellar dimensions in assessment of gestational age in neonates. Radiology 1991;181:581-85

31. Tilea B, Alberti C, Adamsbaum C, et al. Cerebral biometry in fetal magnetic resonance imaging: new reference data. Ultrasound Obstet Gynecol 2009;33:173-81

32. Reichel TF, Ramus RM, Caire JT, et al. Fetal central nervous system biometry on MR imaging. AJR Am J Roentgenol 2003;180:1155-58

33. Hatab MR, Kamourieh SW, Twickler DM. MR volume of the fetal cerebellum in relation to growth. J Magn Reson Imaging 2008;27:840-45

34. Siebert JR, Nyberg DA, Kapur RP. Cerebral mantle thickness: a measurement useful in anatomic diagnosis of fetal ventriculomegaly. Pediatr Dev Pathol $1999 ; 2: 168-75$

35. Loo CK, Freeman B, Quinton A, et al. The significance of cerebral mantle thickness in fetal ventriculomegaly at autopsy. Pathology 2004;36:247-53

36. McLennan JE, Gilles FH, Neff RK. A model of growth of the human fetal brain. In: Gilles FH, Leviton A, Dooling EC, eds. The Developing Human Brain. Boston: John Wright; 1983:43-58

37. Kostovic I, Vasung L. Insights from in vitro fetal magnetic resonance imaging of cerebral development. Semin Perinatol 2009;33:220-33

38. du Plessis AJ. Cerebral blood flow and metabolism in the developing fetus. Clin Perinatol 2009;36:531-48

39. Volpe JJ. Brain injury in premature infants: a complex amalgam of destructive and developmental disturbances. Lancet Neurol 2009;8:110-24

40. Carletti B, Rossi F. Neurogenesis in the cerebellum. Neuroscientist 2008;14:91-100

41. Volpe JJ. Cerebellum of the premature infant: rapidly developing, vulnerable, clinically important. J Child Neurol 2009;24:1085-104 